\title{
SG2IoT - Uma Arquitetura para Integração de Dispositivos Elétricos Inteligentes de Abordagem Legada em Sistemas Smart Grid Baseados na IoT
}

\author{
Wanderson Modesto ${ }^{1}$, Augusto Venâncio Neto ${ }^{1}$, Denis do Rosário ${ }^{2}$, Eduardo Cerqueira ${ }^{2}$ \\ ${ }^{1}$ Universidade Federal do Rio Grande do Norte (UFRN), Natal/RN, Brasil \\ ${ }^{2}$ Universidade Federal do Pará (UFPA), Belém/PA, Brasil \\ wanderson@ppgsc.ufrn.br, augusto@dimap.ufrn.br, \{denis, cerqueira\}@ufpa.br
}

\begin{abstract}
The interoperability between legacy systems and Intelligent Electronic Devices (IED) used by the Smart Grid has become an important issue, since each manufacturer can adopt a different standard. For the integration of legacy IEDs in a Smart Grid environment based on IoT, we introduce the SG2IoT (Legacy Smart Grid to IoT Protocol Integration Approach), which allows the coexistence of multiple IEDs in a scalable and flexible environment made possible by the SG-Cloud-IoT ecosystem. The evaluation of the proposal was carried out through prototyping atop a lab-premised testbed featuring real-world technologies, which aims at validating the proposed architecture and estimate the cost impact of the prototype use.
\end{abstract}

Resumo. A interoperabilidade entre os sistemas e os dispositivos eletrônicos inteligentes (IEDs) legados, utilizados pela Smart Grid, tornou-se uma questão importante, visto que cada fabricante pode adotar um padrão diferente. Para integração de IEDs legados em um ambiente Smart Grid baseados na IoT, introduzimos o SG2IoT (do inglês Legacy Smart Grid to IoT Protocol Integration Approach), que permite a coexistência de múltiplos IEDs em um ambiente escalável e flexível possibilitado pelo ecossistema SG-Cloud-IoT. A avaliação da proposta foi realizada por prototipagem em uma bancada de testes laboratorial com tecnologias reais, visando validação da arquitetura proposta e uma estimativa de impacto no custo pelo seu uso.

\section{Introdução}

As Redes Elétricas Inteligentes (SG, do inglês Smart Grid) foram proposta com a perspectiva de modernizar um sistema crítico essencial (energia) a partir de um ecossistema de Tecnologias da Informação e Comunicação (TIC's) inovadoras e emergentes para a criação de uma rede de distribuição de energia automatizada, inteligente e amplamente distribuída [Fang et al. 2012]. Com o passar dos anos, a lista de tecnologias emergentes foi mudando, permitindo que novas TIC's fossem incorporadas ao ecossistema SG e deixasse de ser tendência, tornando-se realidade. Essa lista de TIC's hoje é encabeçada pelo paradigma da Internet das Coisas (IoT, do inglês Internet of Things), a computação em nuvem (ou em inglês, Cloud Computing) e também pela extensão da nuvem nas premissas da bordas do espaço cibernético, denominada computação nas bordas (do inglês, Edge Computing) [Negash et al. 2018]. A IoT permite que uma grande quantidade de 
dados produzidos pela SG sejam coletados por sensores inteligentes (IEDs, do inglês Intelligent Electronic Device) localizados nas diferentes partes da rede, sejam transmitidos para a central de monitoramento e controle da operadora de energia [Mota et al. 2019]. Essa grande quantidade de dados deve ser processada, analisada e armazenada de maneira econômica, o que impõe requisitos consideravelmente rígidos a serem respeitados [Saleem et al. 2019].

Os sistemas SG modernos, que abrangem um ecossistema de TIC's complexo e incluem IoT, computação em nuvem e na borda são aqui denominados como SG-CloudIoT. A plena realização da SG-Cloud-IoT irá potencialmente estabelecer as bases para benefícios futuros, aproveitando um conjunto de recursos realmente avançados em níveis sem precedentes e permitindo que novas oportunidades surjam no mercado de SG e agreguem valor por meio de inovações inteligentes que, impactem significativamente na otimização de despesas capitais (CAPEX, do inglês CAPital EXpenditure) e operacionais (OPEX, do inglês OPerational EXpenditure). Entretanto, os sistemas legados não podem ser descartados durante a evolução de um sistema elétrico convencional para SG-CloudIoT, pois podem inviabilizar por completo o processo de mudança.

Um protocolo SG é definido como um barramento que define métodos padronizados de comunicações lógicas entre entre IEDs em subestações elétricas, equipamentos de processo primário, dispositivos secundários e Sistemas de Supervisão e Aquisição de Dados (SCADA, do inglês Supervisory Control and Data Acquisition), que coexistem em um mesmo ecossistema do operador de energia. Os principais protocolos legados SG para automação de subestações são o DNP3 [IEEE 2012] e o IEC 61850 [IEC61850 2020], eles estão presente em praticamente todos os sistemas de automação e controle de subestações [Horalek et al. 2013] [Ortega et al. 2016]

A garantia de plena interoperabilidade entre IEDs legados e os demais sistemas de energia se apresenta como desafio constante ao longo de todos esses anos de vida da SG. No escopo dessa pesquisa, partimos do pressuposto que os IEDs legados precisam ser incorporados a IoT para que possam convergir em uma SG-Cloud-IoT, de modo que sejam acessados de forma ubíqua para coleta e análise de dados. A plataforma IoT desempenha papel chave na SG-Cloud-IoT ao trazer os benefícios da Internet aberta a um sistema que, de forma clássica e estratégica (por se tratar de um sistema crítico), é proprietário por definição [Cheruvu. et al. 2020]. APIs estão disponíveis comercialmente para uso em larga escala, como a NGSI que é adotada pela plataforma FIWARE $^{1}$, e orientam suas abordagens para permitir comunicação em toda a IoT.

Para lidar com o desafio supracitado, propomos o SG2IoT (Legacy Smart Grid to IoT Protocol Integration Approach), uma solução holística para o ecossistema SGCloud-IoT que opera de forma distribuída em ambiente edgelcloud com capacidade de automatizar e gerenciar a integração de IEDs legados (IEC 61850 e DNP3) SG na IoT. A arquitetura possui componentes modulares que atuam na borda da rede e possuem capacidade de integrar IEDs legados à arquitetura SG-Cloud-IoT. Além da proposta SG2IoT, este trabalho tem as seguintes contribuições; $(i)$ extensão das capacidades da computação em nuvem às bordas do ecossistema SG-Cloud-IoT, de modo a permitir um ambiente mais distribuído, visando a um sistema bem mais flexível, permitindo latências baixas, análises

\footnotetext{
${ }^{1} \mathrm{https}: / /$ www.fiware.org/
} 
e atuações em premissa local; e (ii) avaliação da proposta por prototipagem em bancada de testes laboratorial composta por dispositivos e tecnologias facilitadoras reais.

O restante deste artigo está organizado como segue. A Seção 2 apresenta um estudo sobre os principais trabalhos relacionados no contexto desta pesquisa. A Seção 3 descreve a proposta SG2IoT desenvolvida neste trabalho. A Seção 4 oferece resultados da prova de conceito por prototipagem sobre uma bancada de testes laboratorial real. Por fim, a Seção 5 conclui o artigo e propõe trabalhos futuros.

\section{Trabalhos Relacionados}

A integração de sistemas legados aos ecossistemas SG é uma demanda constante dos operadores de energia que visam modernizar seus ecossistemas rumo a SG. Tendo como base os desafios associados ao ecossistema SG-Cloud-IoT, este item apresenta o resultado de uma pesquisa tanto no contexto das bases científicas quanto de soluções de mercado. $\mathrm{O}$ escopo das buscas se deu em soluções que visam permitir interoperabilidade de sistemas legados SG ao ambiente Cloud-IoT, considerando integração de IEDs acessíveis a partir dos principais protocolos SG: IEC 61850 e DNP3.

Os autores em [Araújo et al. 2018] propõem um middleware para integrar equipamentos elétricos legados na infraestrutura da SG por meio das redes de sensores sem fio. O middleware é executado em um nó coletor de uma rede de sensores sem fio, baseada em ZigBee, que opera no padrão IEEE 802.15.4, sendo proposto para solucionar problemas relacionados à integração de equipamentos elétricos legados em uma SG que usa um protocolo de comunicação padronizado como: o IEC 61850, o DNP3 ou Modbus. A principal característica deste middleware é a tradução de mensagens do centro de controle da subestação de energia e os equipamentos elétricos da SG. No entanto, o trabalho não faz uso de nenhum dos principais protocolos de transporte usados pela IoT como, por exemplo, o CoAP, MQTT, HTTP e nem faz uso da nuvem ou borda da rede.

No trabalho realizado por [Shin et al. 2017] o modelo da IEC 61850 foi traduzido para o protocolo CoAP. Os autores promovem um mapeamento robusto, porém simples, do CoAP para a IEC 61850 e ainda fazem uma comparação entre o CoAP com os protocolos MQTT e SOAP para demonstrar a validade de utilizá-lo. No entanto, o principal objetivo do trabalho foi demonstrar como o protocolo CoAP, baseado em serviços REST, poderia funcionar em conjunto com o modelo de dados de IEC $61850 \mathrm{em}$ um ambiente SG e não chegou a utilizar a nuvem ou a borda da rede.

O mercado majoritariamente lida com a integração de sistemas legados SG a IoT a partir de uma arquitetura baseada em gateway. O gateway atua na fronteira entre a rede dos sistemas legados e a conexão ao backhaul do operador de energia oferecendo serviço de encapsulamento/tradução de mensagens IEC 61850/DNP3 em pacotes de outra tecnologia protocolar. Empresas como a Cisco, Intel, Dell, entre outras, fornecem gateways IoT inteligentes em escala comercial como, por exemplo, o Dell Edge Gateway. No entanto, esses dispositivos foram fabricados explicitamente para serem gateways IoT, são proprietários e compatíveis apenas com as plataformas IoT em nuvem disponibilizadas pelo fornecedor. Esse tipo de solução acarreta vários problemas em um sistema SG, tais como: parte da infraestrutura de comunicação será de propriedade e gerenciada por terceiros, questões de privacidade, custos na aquisição da solução e aumento da complexidade da SG [Nugur et al. 2019]. 
A partir do estudo realizado sobre os trabalhos relacionados e das soluções propostas pelo mercado, não encontramos soluções disponíveis capazes de integrar diferentes protocolos legados SG a um ambiente SG-Cloud-IoT pleno (considerando protocolos de comunicação IoT e cenário Cloud ou Edge), o que posiciona nossa pesquisa como inovadora e de importante relevância. A seção seguinte descreve em detalhes nossa proposta.

\section{Abordagem/Arquitetura SG2IoT}

O SG2IoT visa permitir o controle e monitoramento de IEDs, de arquitetura legada, que estão presentes em ecossistemas SG-Cloud-IoT. A abordagem do SG2IoT para integrar esses dispositivos físicos aos ecossistemas SG-Cloud-IoT oferece um ambiente mais flexível, monitorável e adaptável para acomodar novos serviços e aplicativos sem causar mudanças significativas no cenário adjacente. A solução é resultado do projeto de pesquisa e desenvolvimento Sistema IoT-Cloud de Medição Centralizada de Energia Voltado a Rede CEA, sob coordenação da UFPA, em parceria com a UFRN.

A arquitetura funcional do SG2IoT como os principais componentes da arquitetura que foram implementados é apresentada na Figura 1 e consiste em um conjunto de componentes responsáveis por realizar a integração dos IEDs com os componentes do ambiente das plataformas IoT que se comunicam uns com os outros e com a nuvem. Tipicamente, os componentes da solução SG2IoT podem ser implantados e distribuídos em diferentes máquinas servidoras, mas, por questão de simplicidade, foram apresentados e implantados em uma única instância.

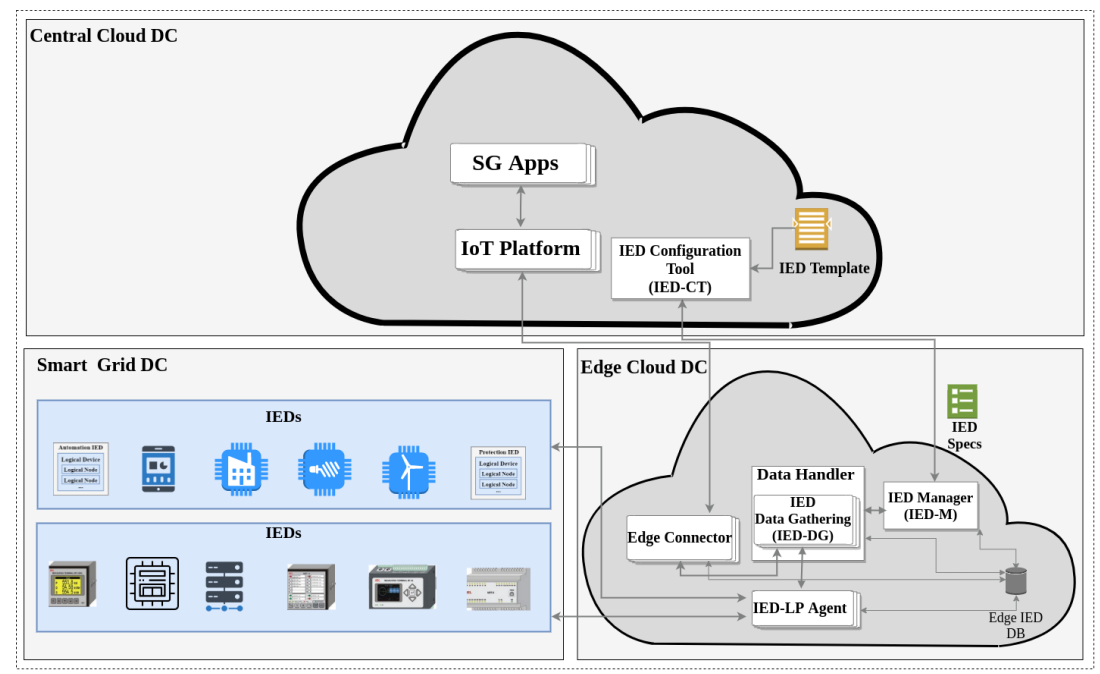

Figura 1. Arquitetura do SG2loT

Os componentes da arquitetura do SG2IoT foram desenvolvidos utilizando a linguagem de programação Python e têm o pressuposto de poderem ser executados tanto em dispositivos que possuem recursos restritos de memória e processamento (como um Raspberry $\mathrm{Pi}$ ) quanto em máquinas com alto poder computacional (como servidores).

O Data Handler, composto por um componente chamado de IED-DG, é o núcleo e componente principal do SG2IoT, possui, direta ou indiretamente, comunicação com todos os outros componentes da arquitetura. O Data Handler recebe os parâmetros e configurações dos dados dos IEDs através do IED-M. 
O IED-LP Agent é usado pelo Data Handler para converter os dados recebidos de um IED conectado. Ele é a implementação de um dos protocolos de comunicação, por exemplo: IEC 61850 e DNP3. Cada IED-LP Agent utiliza bibliotecas de código aberto específicas, como a libiec $61850^{2}$ e a pydnp $3^{3}$, dependendo do dispositivo que ele precise conectar-se. Este módulo realiza as tarefas relacionadas à conversão dos protocolos SG.

O IED-DG mantém as configurações e parâmetros dos dados definidos pelo administrador do sistema. O IED-DG contém informações, como os parâmetros de comunicação que são exigidos pelo IED-LP Agent, quando coletar novos dados dos dispositivos conectados e enviar para as aplicações para visualização ou armazenamento persistente. O IED- $M$ recebe todos os parâmetros de comunicação e registro de dados por meio de um arquivo de configuração (IEDSpecs) que é criado dinamicamente por meio de um arquivo central, o IED Template recebido do IED-CT.

O Edge Connector funciona como um gateway e atua como um gerenciador, intermediando os dados dos dispositivos a serem encaminhados para as aplicações e para as plataformas de IoT, enviando as mensagens provenientes dos dispositivos para as plataformas IoT por protocolos de comunicação como, por exemplo, MQTT ou HTTP. Ele recebe essas informações do IED-DG e as traduz para o formato da plataforma IoT alvo para, em seguida, encaminhá-las. O Edge Connector é o módulo que possibilita a interação com o FIWARE (ou outra plataforma IoT), cria, adiciona dispositivos à nuvem, publica os dados de um dispositivo e atualiza as propriedades dos dispositivos. Ele age como um broker de mensagens fornecendo armazenamento e também processamento de dados, permitindo o acesso de forma local quando a conexão com a nuvem não pode ocorrer.

O IEC-CT é dirigido por template e recebe um arquivo IED Template, configuração usada por ele, que contém a estrutura hierárquica de IED-LP Agent, interpreta o IED Template e realiza as validações iniciais, cria o IEDSpecs e envia as configurações para o IED-M. O IEC-CT possui uma WebUI que é utilizada como uma interface para executar tarefas de gerenciamento como o upload dos arquivos de configuração, o IED Template, e o envio do IEDSpecs para o endereço do IED-M.

O SG2IoT foi idealizado seguindo o modelo cliente/servidor, sendo o SG2IoT o cliente e consumindo dados de vários IEDs, os servidores. Entre outras funcionalidades o SG2IoT realiza o gerenciamento e monitoramento de dispositivos, assim, possibilitando o monitoramento de IEDs por meio de dashboards. A Figura 2 apresenta o diagrama de sequência do workflow dessa funcionalidade.

Como pode ser visto na Figura 2, o workflow dessa funcionalidade parte do IED. Ele envia seus dados para o SG2IoT e o IED-LP Agent, módulo responsável por interpretar e converter os dados recebidos. Em seguida, o IED-LP Agent encaminha os dados para o Data Handler que lê as informações recebidas e as encaminha para o Edge Connector, sendo esse módulo o responsável por estabelecer a conexão com a plataforma FIWARE. Assim que a conexão é estabelecida, ele adiciona o IED como um dispositivo na plataforma IoT. Com o dispositivo criado, a próxima etapa é publicar os dados do IED no FIWARE, o Edge Connector publica os dados do IED na plataforma que notifica a mudança no seu contexto para as aplicações sobrescritas a ela.

\footnotetext{
${ }^{2}$ https://libiec61850.com/libiec61850/

${ }^{3}$ https://github.com/ChargePoint/pydnp3
} 


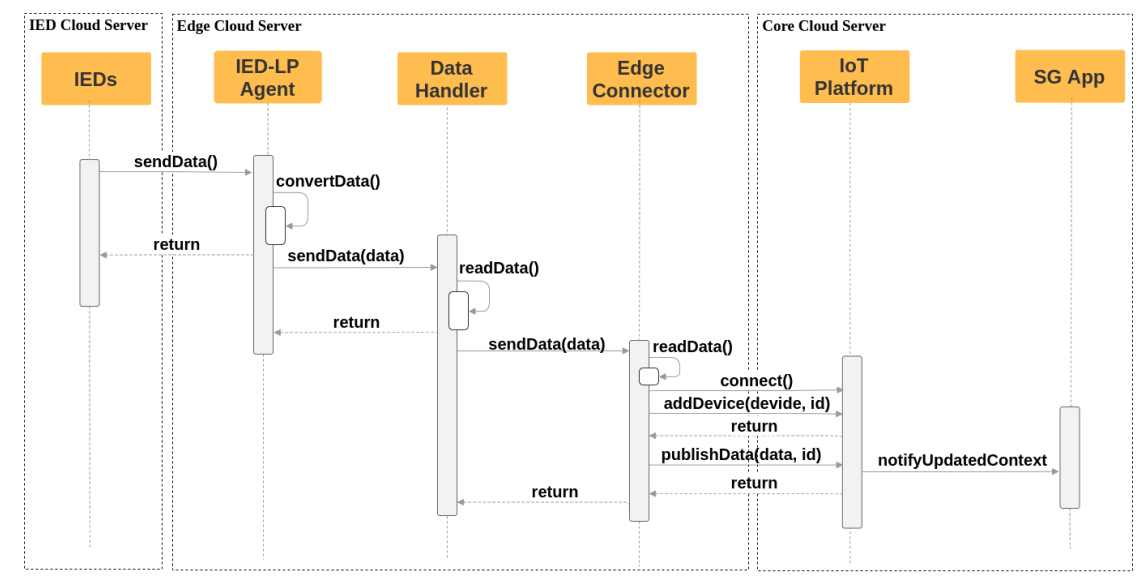

Figura 2. Diagrama de sequência para criação de um dispositivo no SG2loT

\section{Prova de Conceito}

Para avaliar a proposta SG2IoT, desenvolvemos uma bancada de testes laboratorial composta por dispositivos e tecnologias facilitadores reais de uma SG-Cloud-IoT. Além de um protótipo do SG2IoT, desenvolvemos uma aplicação de monitoramento de dados dos IEDs que opera sobre a plataforma FIWARE para permitir um ambiente compatível com medição de energia inteligente em tempo real. A representação dos IEDs se deu a partir do uso de dados sintéticos em datasets simulados pela impossibilidade de aquisição de equipamentos nas tecnologias indicadas. Os IEDs foram modelados como entidades de contexto no componente Orion do FIWARE, que se sobrescrevem primeiramente para então ter suas medições consumidas, em tempo real, pela aplicações de medição de energia inteligente.

Foram utilizados 3 (três) máquinas virtuais que rodam o sistema operacional Ubuntu 18.04 e se comunicam através da rede local cabeada, como forma de oferecer um ambiente de conectividade que abarque alta densidade de mensagens simultâneas provenientes de múltiplos IEDs. As máquinas virtuais utilizadas no experimento foram criadas em um ambiente de computação em nuvem disponível na bancada de testes. A Figura 3 apresenta o layout da bancada de testes laboratoriais e seus principais componentes.

Como esboça a Figura 3, a máquina (A) abriga a execução de diferentes IEDs, sendo provisionada com 8 vCPUs e 10 GB de memória RAM. Já a máquina (B) roda a plataforma FIWARE e tem provisionamento de 8 vCPUs e 16 GB de memória RAM. O FIWARE participa da bancada de testes a partir dos seus seguintes componentes fundamentais: o Orion Context Broker, IoT Agent para MQTT e HTTP, o Cygnus e um banco de dados MySQL. Por fim, a máquina (C) é o ambiente de execução do protótipo SG2IoT, e tem alocado 10 vCPUs e 16 GB de memória RAM. O SG2IoT foi executado em um container Docker que teve seu uso de memória RAM restrito a 2 GB e poder de processamento para $2 \mathrm{vCPU}$.

Além do protótipo SG2IoT e da aplicação de medição inteligente, desenvolvemos geradores de tráfegos IEC61850 e DNP3 para simular parâmetros elétricos compatíveis com IEDs de medição inteligente, tais como: tensões e correntes das fases, data e hora, potências ativas e reativas das fases, frequência, entre outros. Para definir a capacidade máxima do protótipo, o mesmo foi submetido a vários níveis de estresse a partir do au- 


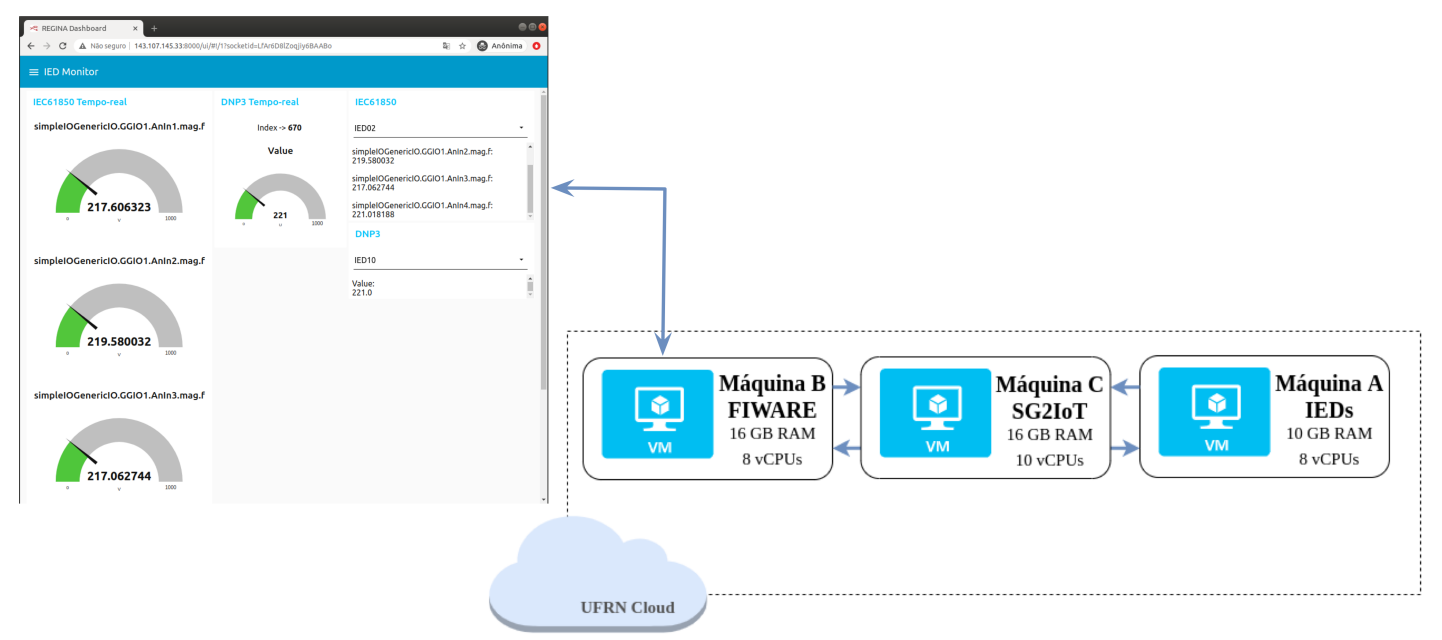

Figura 3. Layout da bancada de testes laboratorial

mento da escala de IEDs simultaneamente ativados na bancada de testes laboratorial. Identificamos que a densidade máxima de IEDs no testbed foi de 100 dispositivos ativos, uma vez que qualquer valor acima deste faz com o contêiner que abriga o SG2IoT reinicie por esgotamento de memória RAM, e a bancada de testes torna-se inoperante.

Realizamos 4 grupos de experimentos para cada um dos dois protocolos, diferenciados pela variação da densidade de IEDS (nomeadamente 1, 10, 50 e 100 tanto do tipo IEC 61580 quanto DNP3), com threads simultâneas realizando solicitações ininterruptas (1 mensagem a cada segundo por IED) ao SG2IoT durante o período de 1 hora. Os experimentos foram repetidos 10 vezes cada em condições idênticas, de forma a obter um intervalo de confiança de $95 \%$.

O primeiro experimento considera a subscrição da aplicação IoT a um grupo de IEDs legados ativos (IEC 61850 e DNP3) para receber medições em tempo real. A Figura 3 apresenta um screenshot da Web dashboard da aplicação IoT de monitoramento inteligente em execução real. É possível perceber que que as medições originadas por IEDs legados são apresentadas pela aplicação IoT em tempo real, a partir da integração realizada pelo SG2IoT. Para verificar a capacidade do SG2IoT em integrar IEDs legados à SG-Cloud-IoT de forma holística, monitoramos a vazão média gerada pelos IEDs ativos nos diferentes experimentos realizados (apresentada pela Figura 4).

Como se pode observar na Figura 4, o protótipo SG2IoT recebe os pacotes publicados pelos IEDs legados em seus respectivos formatos (Figura (4a)), converte os dados recebidos para a semântica compreensível do IoT Agent e em seguida encaminha as mensagens a plataforma IoT através do protocolo MQTT (Figura (4b)). Esta prova de conceito confirma que a proposta SG2IoT é funcional, que seus componentes estão em conformidade com a arquitetura proposta, e que as interfaces internas e externas da arquitetura foram validadas com sucesso. Além disso, os resultados comprovam a eficácia do SG2IoT em compatibilizar de forma holística IEDs legados à SG-Cloud-IoT.

Visto que o ecossistema proposto oferece a perspectiva de automatizar a conexão de IEDs de diferentes protocolos à SG-Cloud-IoT, realizamos um outro experimento para estimar o custo de operação do protótipo no testbed. Para tal, aferimos o impacto do uso 


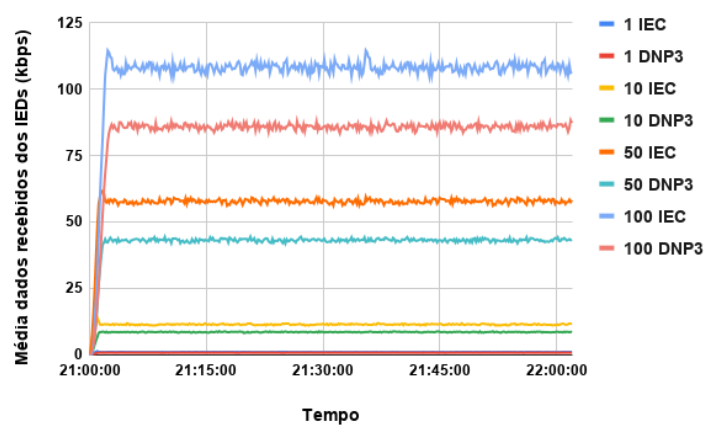

(a) Dados recebidos pelo SG2IoT

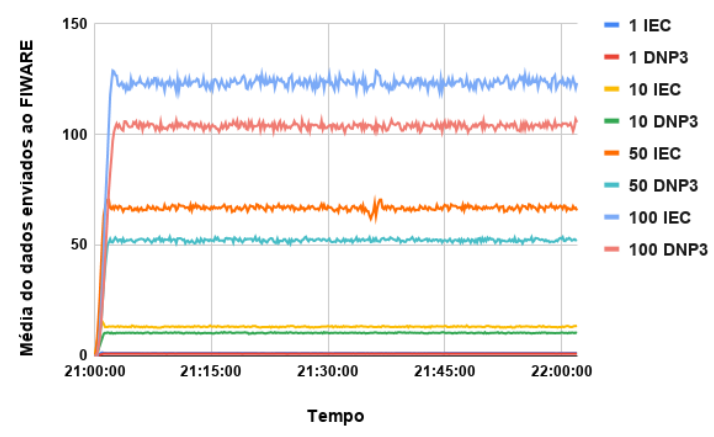

(b) Dados enviados pelo SG2IoT à plataforma FIWARE

Figura 4. Média de dados recebidos pelo SG2IOT e enviados para a plataforma FIWARE

do protótipo no consumo de CPU e memória RAM pela variação da densidade de IEDs no testbed. Tais métricas foram coletadas a partir da ferramenta Netdata ${ }^{4}$. Além disso, analisamos também o tempo de resposta do protótipo nos experimentos como forma de estimar sua escalabilidade. Para tal, implementamos diretamente no protótipo SG2IoT funções que registram o instante da execução e conclusão de cada tarefa. Os resultados desse experimento são apresentados na Figura 5.

As Figuras 5b e 5a apresentam o comportamento médio do consumo de CPU e memória RAM nos experimentos realizados com IEDs em diferentes granularidades e para cada protocolo legado, considerando o testbed provisionado com 2 GB de RAM e 2 vCPU. Segundo a 5a, o consumo de memória RAM é idêntico para os dois protocolos nos experimentos com 1, 10 e 50 IEDs, apresentando uma baixa variação de 15,10 MB no experimento com 100 IEDs. A partir dos resultados da Figura 5b, podemos perceber que o SG2IoT impactou no mesmo consumo de CPU para todos os experimentos $(1,10$, 50 e 100 IEDs).

Finalmente, a Figura 5c apresenta os tempos de resposta médios que o protótipo SG2IoT impactou nos experimentos realizados. O tempo de resposta fim-a-fim foi medido pela diferença de tempo a partir do momento em que o remetente (IED) coloca o conteúdo de dados no topo de sua pilha de transmissão até o momento em que o receptor (FIWARE, por meio do Orion Context Broker) extrai os dados de sua pilha de transmissão e confirma o registro da informação. Para sincronizar os relógios de todas as máquinas do testbed, utilizamos um servidor NTP (do inglês Network Time Protocol) disponível localmente. Os tempos de resposta foram decompostos em duas partes: (i) SG2ToT, tempo de resposta médio para receber as mensagens IEDs em formato SG legado, converter em MQTT e encaminhar a plataforma IoT; e (ii) FIWARE, tempo de resposta médio da plataforma IoT para receber a medição IED e entregar a aplicação de medição de energia inteligente.

Como pode ser visto na Figura 5c, o protótipo SG2ToT apresenta tempo de resposta relativamente estável para tratar as mensagens dos diferentes IEDs. Já a plataforma IoT apresenta tempo de resposta bastante superior ao SG2IoT (em média 64,88\%), com

\footnotetext{
${ }^{4}$ http://www.netdata.cloud
} 


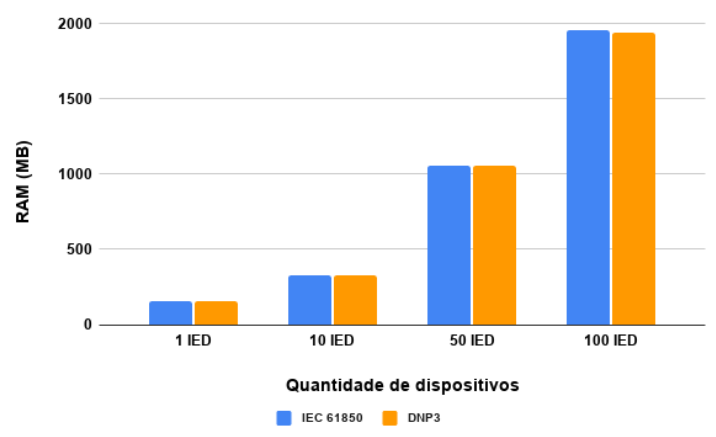

(a) Memória RAM

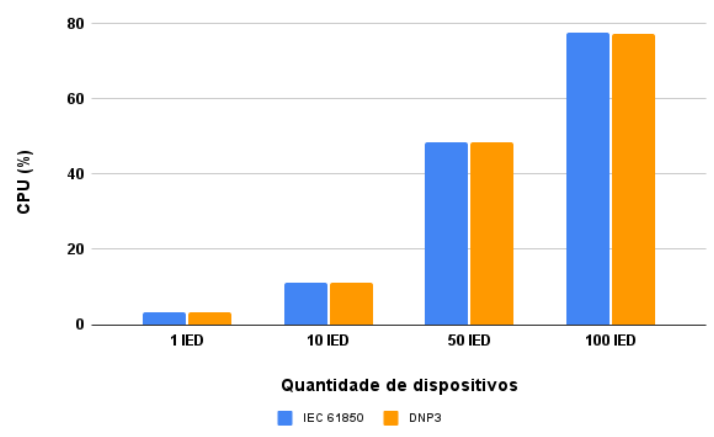

(b) CPU

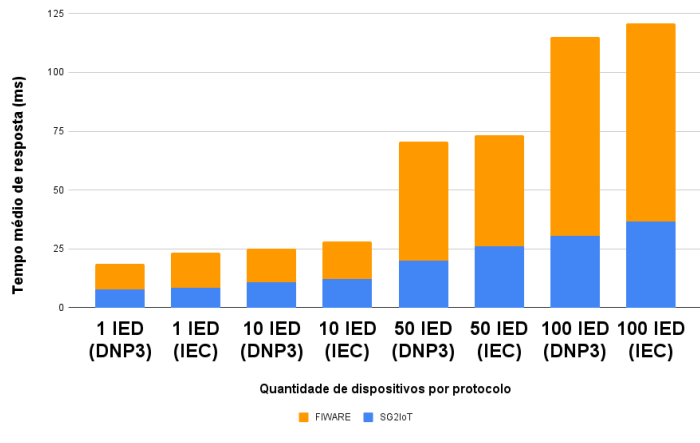

(c) Tempo de Resposta

Figura 5. Custo do protótipo a partir do impacto de seu uso na bancada de testes

taxa de crescimento exponencial a medida que mais IEDs são ativados. A explicação para isso está na complexidade arquitetural do FIWARE e seu fluxo de trabalho extenso em comparação ao SG2IoT, o que tornou o gargalo computacional no testbed. Apesar disso, os resultados indicam que o protótipo ficou dentro dos padrões exigidos pelas aplicações SG, $200 \mathrm{~ms}$ de acordo com [Kuzlu and Manisa 2013], bem como foi capaz de atender aos requisitos de desempenho do tempo médio de resposta, $500 \mathrm{~ms}$ de acordo com o que é definido pela norma do IEC 61850 [IEC61850 2020].

\section{Conclusão}

Este artigo apresentou o SG2IoT, uma solução distribuída em ambiente edge/cloud para integração automatizada de IEDs baseados em protocolos legados IEC 61850 e DNP3 a um ecossistema SG-Cloud-IoT. O SG2IoT oferece um ambiente flexível, monitorável e automatizado na infraestrutura para acomodar novas aplicações e serviços. Para realizar prova de conceito da solução proposta, um protótipo foi construído sobre uma bancada de testes real compatível com a SG-Cloud-IoT. Os testes sugerem a eficácia do SG2IoT em integrar IEDs legados a prova SG-Cloud-IoT, com estimativa de baixo custo computacional. Além disso, o tempo de resposta impactado nos experimentos sugerem também um bom nível de escalabilidade. Como trabalho futuro, iremos aferir o desempenho do SG2IoT, visando introspecções mais abrangentes e identificar melhorias na solução. 


\section{Agradecimentos}

A CAPES e o projeto Sistema IoT-Cloud de Medição Centralizada de Energia Voltado a Rede CEA fomentaram este trabalho.

\section{Referências}

Araújo, P. R. C., Filho, R. H., Rodrigues, J. J., Oliveira, J. P., and Braga, S. A. (2018). Middleware for integration of legacy electrical equipment into smart grid infrastructure using wireless sensor networks. International Journal of Communication Systems, 31(1):e3380.

Cheruvu., S., A., K., N., S., and D.M, W. (2020). IoT Frameworks and Complexity.

Fang, X., Misra, S., Xue, G., and Yang, D. (2012). Smart grid-the new and improved power grid: A survey. IEEE communications surveys \& tutorials, 14(4):944-980.

Horalek, J., Matyska, J., and Sobeslav, V. (2013). Communication protocols in substation automation and iec 61850 based proposal. In 2013 IEEE 14th International Symposium on Computational Intelligence and Informatics (CINTI), pages 321-326. IEEE.

IEC61850 (2020). International electrotechnical commission standard - iec61850. Disponível em: https://www.iec.ch/smartgrid/standards/.

IEEE (2012). 1815-2012 - ieee standard for electric power systems communicationsdistributed network protocol (dnp3). Disponível em: https://standards . ieee.org/content/ieee-standards/en/standard/1815-2012. html.

Kuzlu, M. and Manisa, P. (2013). Assessment of communication technologies and network requirements for different smart grid applications. In 2013 IEEE PES Innovative Smart Grid Technologies Conference (ISGT), pages 1-6.

Mota, R., Riker, A., and Rosário, D. (2019). Adjusting group communication in dense internet of things networks with heterogeneous energy sources. In Anais do XI Simposio Brasileiro de Computacao Ubiqua e Pervasiva. SBC.

Negash, B., Rahmani, A. M., Liljeberg, P., and Jantsch, A. (2018). Fog Computing Fundamentals in the Internet-of-Things, pages 3-13. Springer International Publishing.

Nugur, A., Pipattanasomporn, M., Kuzlu, M., and Rahman, S. (2019). Design and development of an iot gateway for smart building applications. IEEE Internet of Things Journal, 6(5):9020-9029.

Ortega, A., Schweitzer, C. M., Shinoda, A. A., and Ortega, A. V. (2016). Simulation of the dnp3 protocol over tcp/ip on a network ieee $802.11 \mathrm{~g}$ ad-hoc with smart meter. In 2016 IEEE ANDESCON, pages 1-4.

Saleem, Y., Crespi, N., Rehmani, M. H., and Copeland, R. (2019). Internet of things-aided smart grid: technologies, architectures, applications, prototypes, and future research directions. IEEE Access, 7:62962-63003.

Shin, I.-J., Song, B.-K., and Eom, D.-S. (2017). International electronical committee (iec) 61850 mapping with constrained application protocol (coap) in smart grids based european telecommunications standard institute machine-to-machine $(\mathrm{m} 2 \mathrm{~m})$ environment. Energies, 10(3):393. 